\title{
Bioconversion of FR901459, a novel derivative of cyclosporin A, by Lentzea sp. 7887
}

\author{
Satoshi Sasamura ${ }^{1,4}$, Motoo Kobayashi ${ }^{1}$, Hideyuki Muramatsu ${ }^{2}$, Seiji Yoshimura ${ }^{1}$, Takayoshi Kinoshita ${ }^{1,5}$, \\ Hidenori Ohki ${ }^{1,6}$, Kazuki Okada ${ }^{3}$, Yoko Deai ${ }^{1}$, Yukiko Yamagishi ${ }^{1}$ and Michizane Hashimoto ${ }^{2,4}$
}

FR901459, a product of the fungus Stachybotrys chartarum No. 19392, is a derivative of cyclosporin A (CsA) and a powerful immunosuppressant that binds cyclophilin. Recently, it was reported that CsA was effective against hepatitis C virus (HCV). However, FR901459 lacks active moieties, which are essential for synthesizing more potent and safer derivatives of this anti-HCV agent. Here we identified an actinomycete strain (designated 7887) that was capable of efficient bioconversion of FR901459. Structural elucidation of the isolated bioconversion products (1-7) revealed that compounds 1-4 were mono-hydroxylated at the position of 1-MeBmt or 9-MeLeu, whereas compounds 5-7 were bis-hydroxylated at both positions. The results of morphological and chemical characterization, as well as phylogenetic analysis of 16S ribosomal DNA (rDNA), suggested that strain 7887 belonged to the genus Lentzea. Comparison of the FR901459 conversion activity of strain 7887 with several other Lentzea strains revealed that although all examined strains metabolized FR901459, strain 7887 had a characteristic profile with respect to bioconversion products. Taken together, these findings suggest that strain 7887 can be used to derivative FR901459 to produce a chemical template for further chemical modifications that may provide more effective and safer anti-HCV drugs.

The Journal of Antibiotics (2015) 68, 511-520; doi:10.1038/ja.2015.19; published online 18 March 2015

\section{INTRODUCTION}

The process of bioconversion, which is also referred to as biotransformation, involves the modification of natural compounds by microorganisms. For example, the anti-hyperlipidemia drug pravastatin is produced on an industrial scale using Streptomyces carbophilus to hydroxylate the compound ML-236B. ${ }^{1}$ In addition, the precursor of anti-fungal drug micafungin is produced via the microbial deacylation of an acyl chain in the lipopeptide FR901379. ${ }^{2}$ As microorganisms are also capable of hydroxylating a wide range of compounds, ${ }^{3-7}$ bioconversion is considered to be a powerful tool for the modification of useful natural products with complicated structures.

Cyclosporin A (CsA) is a cyclic peptide that inhibits cellular cyclophilins and was developed for clinical use as an immunosuppressant critical for organ transplants. The inhibitory activity of CsA is dependent on its binding to cyclophilin, as the resulting complexes inhibit the function of calcineurin in T cells. ${ }^{8}$ Recently, it was reported that CsA suppresses replication of the hepatitis C virus (HCV) genome, and that the inhibition of cyclophilins are critical for $\mathrm{HCV}$ replication. ${ }^{9,10}$ As CsA treatment in combination with interferon was effective against $\mathrm{HCV},{ }^{11}$ a number of CsA derivatives, including DEBIO-025, SCY-635 and NIM-811,12 are currently being evaluated in clinical trials as anti-HCV drugs.
Compound FR901459 is a member of the cyclosporin family and was first identified in the fermentation broth of Stachybotrys chartarum No. $19392 .{ }^{13}$ This compound exerts immunosuppressive activity upon binding to cyclophilin and also has potent neuroprotective effects by inhibiting mitochondrial damage, ${ }^{14}$ but is structurally distinct from CsA (Figure 1). This compound is considered to have an attractive backbone structure for the development of an anti-HCV drug, but lacks active moieties, which are essential for synthesizing derivatives of this compound. In the present work, we describe the biotransformation, isolation and physicochemical characterization of seven bioconversion products (1-7) of FR901459 that were derived using an actinomycete strain, designated 7887 , which was revealed as a species of Lentzea based on phylogenetic analysis.

\section{RESULTS}

Isolation of bioconversion products (compounds 1-7) from strain 7887

A screening for actinomycete strains with bioconversion activity was performed with 30 strains isolated from soil samples. We identified one strain, designated 7887, that converted FR901459 to a number of derivatized products. To isolate the bioconversion products, strain 7887 was incubated in the presence of $0.5 \mathrm{mg} \mathrm{ml}^{-1}$ FR901459 for

${ }^{1}$ Drug Discovery Research, Astellas Pharma Inc., Tsukuba, Ibaraki, Japan; ${ }^{2}$ Fermentation Research Division, Astellas Research Technologies Co., Ltd, Tsukuba, Ibaraki, Japan and ${ }^{3}$ Intellectual Property, Astellas Pharma Inc., Chuo, Tokyo, Japan

${ }^{4}$ Current address: Preclinical Research Division, Taiho Pharmaceutical Co., Ltd, Tsukuba, Ibaraki, Japan

${ }^{5}$ Current address: Graduate School of Science, Osaka Prefecture University, Sakai, Osaka, Japan

${ }^{6}$ Current address: Business Development Division, Towa Pharmaceutical Co., Ltd, Kadoma, Osaka, Japan

Correspondence: Dr S Sasamura, Preclinical Research Division, Taiho Pharmaceutical Co., Ltd., 3 Okubo, Tsukuba, Ibaraki 300-2611, Japan.

E-mail: s-sasamura@taiho.co.jp

Received 17 September 2014; revised 5 December 2014; accepted 2 February 2015; published online 18 March 2015 


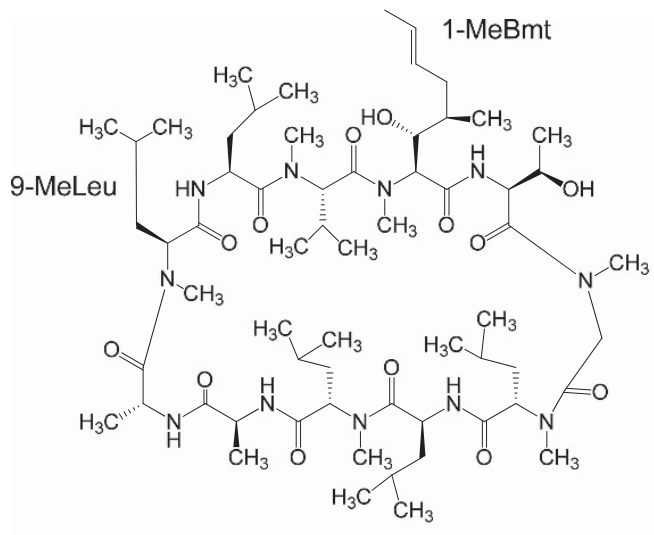

FR901459

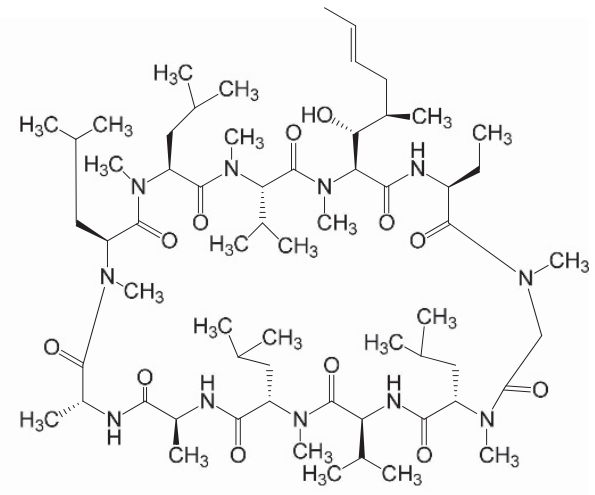

Cyclosporin A

Figure 1 Structures of FR901459 and cyclosporin A. Bmt, (4R)-4-[(E)-2-butenyl]-4-methyl-L-threonine; Me, methyl.

$13 \mathrm{~h}$, and the reaction mixture was extracted with an equal volume of acetone at room temperature. The mixture was filtered with an aid of diatomaceous earth and the diluted filtrate was passed through a 14-1 Diaion HP-20 column (Mitsubishi Chemical, Tokyo, Japan). The column was washed with $25 \%$ aqueous acetone, eluted with methanol and the active fraction was passed through an 8-1 Daisogel SP-120ODS-B column $(15 / 30 \mu \mathrm{m}$, Daiso, Osaka, Japan). After eluting the column with 50 and $60 \%$ aqueous acetonitrile, the following three fractions containing a total of seven compounds were obtained: fractions I (compound 1, 60\% aqueous acetonitrile), II (compounds 2-4, 60\% aqueous acetonitrile) and III (compounds 5 and 7, 50\% acetonitrile).

To isolate the bioconversion products from fraction I, the sample was diluted with an equal volume of water and then passed through a 2-1 Daisogel SP-120-ODS-B column. The column was eluted with $60 \%$ aqueous acetonitrile and the eluate was rechromatographed as above. The active fraction was again passed through a 2-1 Daisogel SP-120ODS-B column and eluted with $70 \%$ aqueous acetonitrile containing $0.1 \%$ trifluoroacetic acid. The active fraction was diluted with an equal volume of water, passed through a 2-l Daisogel SP-120-ODS-B column and eluted with ethyl acetate. The solvent fraction was concentrated to dryness in vacuo, dissolved in $\mathrm{CHCl}_{3}$ and applied to silica gel column (Silica Gel $60 \mathrm{~N}$, spherical, neutral, 40-100 $\mu \mathrm{m}$, Kanto Chemical Co., Inc., Tokyo, Japan). The column was washed with $2 \% \mathrm{MeOH}$ in $\mathrm{CHCl}_{3}$, eluted with 3 and $5 \% \mathrm{MeOH}$ in $\mathrm{CHCl}_{3}$, and the active fraction was concentrated to dryness in vacuo and then precipitated with $\mathrm{n}$-hexane to give $3.7 \mathrm{~g}$ of compound $\mathbf{1}$ as a white powder.

A similar approach was used to isolate the bioconversion products of FR901459 in fractions II and III. The water-diluted fraction II was passed through an 8-1 Daisogel SP-120-ODS-B column, which was then eluted with $60 \%$ aqueous acetonitrile. The active fraction was diluted with an equal volume of water and passed through a 2-1 Daisogel SP-120-ODS-B column. After washing the column with water and adding ethyl acetate, the eluted solvent fraction was concentrated to dryness in vacuo and precipitated by n-hexane to give $32.8 \mathrm{~g}$ of compound 2 as a white powder. The first elution of the ODS column with $60 \%$ aqueous acetonitrile gave a subfraction that contained compounds 3 and 4 . To separate compounds 3 and $\mathbf{4}$, this diluted subfraction was passed through a 2-1 Daisogel SP-120-ODS-B column, and was then eluted with $60 \%$ aqueous acetonitrile. The active fraction of compound 3 was diluted with an equal volume of water, passed through a 1-1 Daisogel SP-120-ODS-B column and then eluted with $60 \%$ aqueous acetonitrile. The active fraction was concentrated to dryness in vacuo to give $322 \mathrm{mg}$ of compound 3 as a pale yellow powder. Using the same purification method as described above for compound 3,740 $\mathrm{mg}$ of compound 4 as a pale yellow powder was obtained.

Similarly, fraction III was diluted with an equal volume of water and passed through an 8-1 Daisogel SP-120-ODS-B column. After eluting the column with $50 \%$ aqueous acetonitrile, the active fractions containing compound 5 or 7 were concentrated in vacuo and then extracted with ethyl acetate. To purify compound $\mathbf{5}$, the solvent extract was concentrated in vacuo, dissolved in $\mathrm{CHCl}_{3}$ and applied to a silica gel column, which was then washed with $3 \% \mathrm{MeOH}$ in $\mathrm{CHCl}_{3}$ and eluted with 4 and $5 \% \mathrm{MeOH}$ in $\mathrm{CHCl}_{3}$. The active fraction was concentrated in vacuo, dissolved in $\mathrm{MeOH}$ and then loaded onto a preparative HPLC column (Mightysil RP-18 GP $250 \mathrm{~mm} \times 20 \mathrm{~mm} \mathrm{I}$. D., $5 \mu \mathrm{m}$; Kanto Chemical Co., Inc.). The column was eluted with $50 \%$ aqueous acetonitrile containing $0.1 \%$ trifluoroacetic acid at a flow rate of $10 \mathrm{ml} \mathrm{min}^{-1}$. The diluted active fraction was passed through a 19-ml Daisogel SP-120-ODS-B column. After the column was washed with water and eluted with ethyl acetate, the solvent fraction was concentrated to dryness in vacuo and precipitated with n-hexane to give $205 \mathrm{mg}$ of compound $\mathbf{5}$ as a white powder. Using the same purification method as described above for compound 5, $227 \mathrm{mg}$ of compound 7 as a white powder was obtained.

To purify compound $\mathbf{6}$, a part of the filtrate from the reaction mixture was diluted with an equal volume of water, passed through a 2-1 Daisogel SP-120-ODS-B column and eluted with 50\% aqueous acetonitrile. The active fraction was diluted with an equal volume of water, passed through a 2-1 Daisogel SP-120-ODS-B column and eluted with $45 \%$ aqueous acetonitrile containing $0.1 \%$ trifluoroacetic acid. The active fraction was diluted with an equal volume of water, passed through a 2-1 Daisogel SP-120-ODS-B column and eluted with ethyl acetate after the column was washed with water. The solvent fraction was concentrated to dryness in vacuo and precipitated with $\mathrm{n}$-hexane to give $1.3 \mathrm{~g}$ of compound $\mathbf{6}$ as a pale yellow powder. The isolation procedures for compounds 1-7 are summarized in Figure 2.

\section{Structure elucidation}

The physicochemical properties of the isolated bioconversion products are summarized in Table 1. All compounds were soluble in $\mathrm{CHCl}_{3}$, ethyl acetate, acetone, dimethyl sulfoxide (DMSO), pyridine and $\mathrm{MeOH}$, and were slightly soluble in water, but were insoluble in n-hexane. The ESI-TOF (time-of-flight) MS analysis (Table 1) showed 


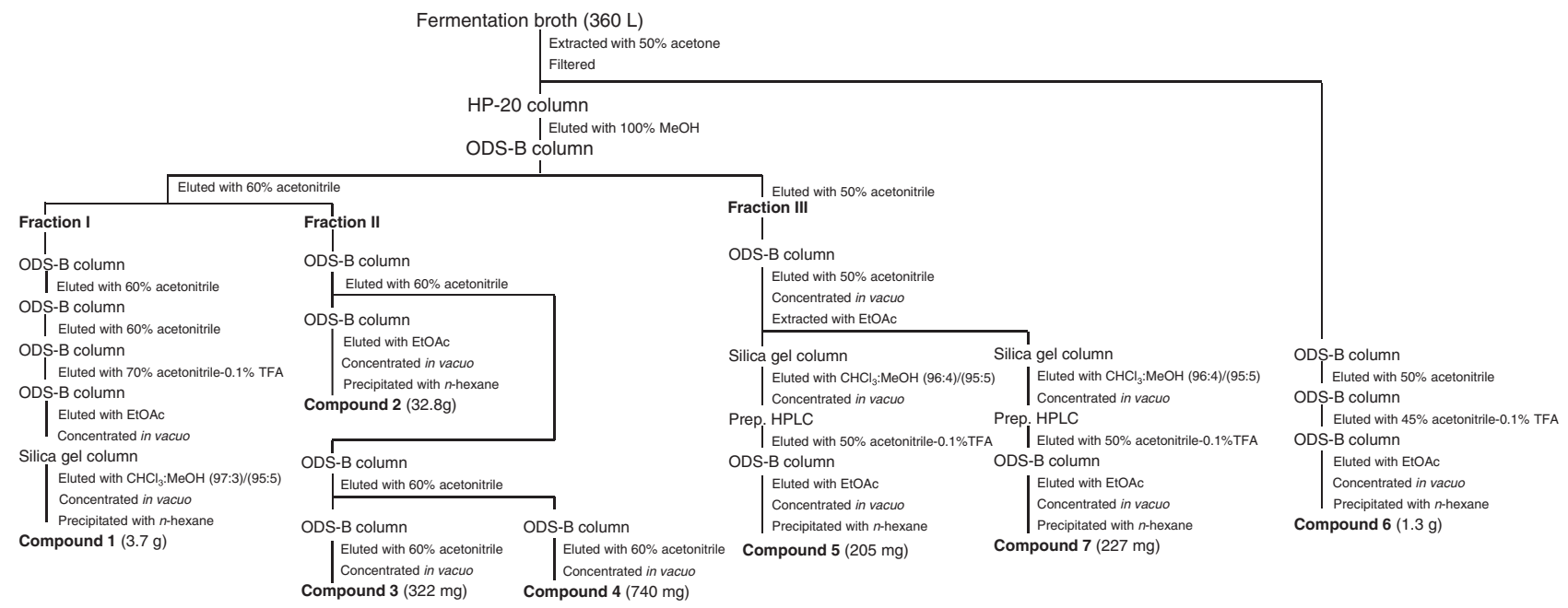

Figure 2 Isolation and purification of compounds 1-7.

each molecular formula for compounds 1-4 to be $\mathrm{C}_{62} \mathrm{H}_{111} \mathrm{~N}_{11} \mathrm{O}_{14}$, and $\mathrm{C}_{62} \mathrm{H}_{111} \mathrm{~N}_{11} \mathrm{O}_{15}$ for compounds 5-7, indicating that compounds 1-4 were mono-hydoxylated forms of FR901459, whereas compounds 5-7 were bis-hydoxylated.

For the NMR study of compounds $1-7$, pyridine- $\mathrm{d}_{5}$ was used as a solvent because it resulted in the best separation of all tested solvents. At least two conformers were observed for all compounds as in the case of FR901459. ${ }^{13}$ By observing ${ }^{13} \mathrm{C}$ NMR and HSQC spectra, the existence of 62 carbons was confirmed, including 11 carbonyl carbons, $11 \mathrm{C} \alpha$ carbons and six N-methyl carbons for all compounds. Five NH protons were indicated by correlation with each adjacent $\mathrm{C} \alpha$ proton in the COSY spectra. The structure of each amino-acid residue was elucidated by the combination of NMR spectra, COSY, TOCSY and HMBC analyses. The peptide sequences were determined to be unchanged from FR901459 by analyzing multiple correlations in the HMBC spectra from $\mathrm{C} \alpha, \mathrm{NH}$ and $\mathrm{N}$-methyl protons to carbonyl carbons.

NMR assignments are shown in the Table 2. Hydroxylated positions were clearly detected by chemical shifts of the terminal methylene ( $\delta_{\mathrm{H}} 4.35$ p.p.m. and $\delta_{\mathrm{C}}$ 63.1p.p.m.) of 1-MeBmt for compound 1, the terminal methylene of 9-MeLeu $\left(\delta_{\mathrm{H}} 3.77\right.$ and 3.71p.p.m., and $\delta_{\mathrm{C}}$ 67.9 p.p.m.) for compound 2, the quaternary carbon of 9-MeLeu ( $\delta_{\mathrm{C}}$ 69.0 p.p.m.) for compound 3 and the terminal methylene of 9-MeLeu $\left(\delta_{\mathrm{H}} 3.91\right.$ and 3.79 p.p.m., and $\delta_{\mathrm{C}} 66.1$ p.p.m. $)$ for compound 4. Oxygenated positions of compounds 6, 5 and 7 were concluded to be combinations of compounds 2, 3 and 4 with compound $\mathbf{1}$ as they have both hydroxylated carbons resonating at 63.1 p.p.m. of 1-MeBmt, and 67.9, 69.0 and 66.1p.p.m. of 9-MeLeu, respectively (Table 2 ).

During the attempted derivatization of these bioconverted products, we obtained FR310363, a tert-butyldimethylsilyl-protected compound in 2-Thr, which was determined to be a crystalline derivative of compound 2. A single crystal of FR310363 was obtained from a solution of ethyl acetate, and X-ray crystallography analysis allowed assignment of the absolute configuration of 4 -MeLeu to be $(S)$. The $\mathrm{X}$-ray structure analysis data have been deposited at the Cambridge Crystallographic Data Centre and allocated the deposition number CCDC 1048671. An ORTEP drawing is shown in Figure 3. We speculated that the absolute configuration of the same position of compound 6 to be $(S)$, and those of compounds 4 and 7 to be $(R)$ based on their ${ }^{13} \mathrm{C}$ NMR similarity. The proposed gross structures of compounds 1-7 are shown in Figure 4.

\section{Biological activity}

Compounds 1-4 inhibited the replication of HCV subgenomic replicons in $\# 50-1$ cells with $\mathrm{IC}_{50}$ values of 1.5, 2.6, 0.47 and $1.0 \mu \mathrm{M}$, respectively. FR901459 also exerted an inhibitory effect with an $\mathrm{IC}_{50}$ value of $0.14 \mu \mathrm{M}$. In contrast, no obvious inhibitory effects were observed for compounds $5-7$ at $10 \mu \mathrm{M}$.

\section{Taxonomic characterization of strain $\mathbf{7 8 8 7}$}

This strain formed compact colonies on the agar surface and had well developed and irregularly branched substrate mycelia that penetrated the agar surface. The cultural characteristics of strain 7887 are summarized in Table 3. Aerial mycelia were moderately developed on 1/5 yeast extract-starch agar, glycerol-asparagine agar (ISP-5), tyrosine agar (ISP-7), Czapek agar, yeast-starch agar and glucoseasparagine agar, and were fragmented into rod-shaped elements. Sclerotic granules, sporangia and motile spores or fragments were not observed on any of the examined media.

The reverse side of colonies appeared reddish brown to pale orange. No melanoid pigments were produced in tryptone-yeast extract broth or peptone-yeast extract-iron agar (ISP-6). Light orange to brown soluble pigments were produced by colonies cultured on yeast extractmalt extract agar (ISP-2), oatmeal agar (ISP-3), inorganic salts-starch agar (ISP-4), glycerol-asparagine agar (ISP-5), tyrosine agar (ISP-7), Bennett's agar, Czapek agar, yeast-starch agar, glucose-asparagine agar and sucrose-nitrate agar. The color of mycelia and soluble pigments were not $\mathrm{pH}$ sensitive.

The physiological characteristics of strain 7887 are summarized in Table 4. L-arabinose, D-xylose, D-glucose, D-fructose, sucrose, inositol, D-mannitol, cellobiose, dextrin, D-galactose, glycerol, D-mannose, maltose, D-melibiose, soluble starch and trehalose were all utilized as carbon sources, but L-rhamnose, raffinose, adonitol, dextran, dulcitol, inulin, lactose, D-melezitose, sorbitol, L-sorbose and xylitol were not utilized. Strain 7887 was able to grow in the temperature range of $10.5-32.5^{\circ} \mathrm{C}$ and exhibited optimal growth at $31.0^{\circ} \mathrm{C}$. Mesodiaminopimelic acid was detected in whole-cell hydrolysates.

Comparison of $16 \mathrm{~S}$ ribosomal DNA (rDNA) sequences showed that strain 7887 had the highest similarity with Lentzea violacea LM $036^{\mathrm{T}}$ (99.2\%). The similarity values of $16 \mathrm{~S}$ rDNA sequences between strain 


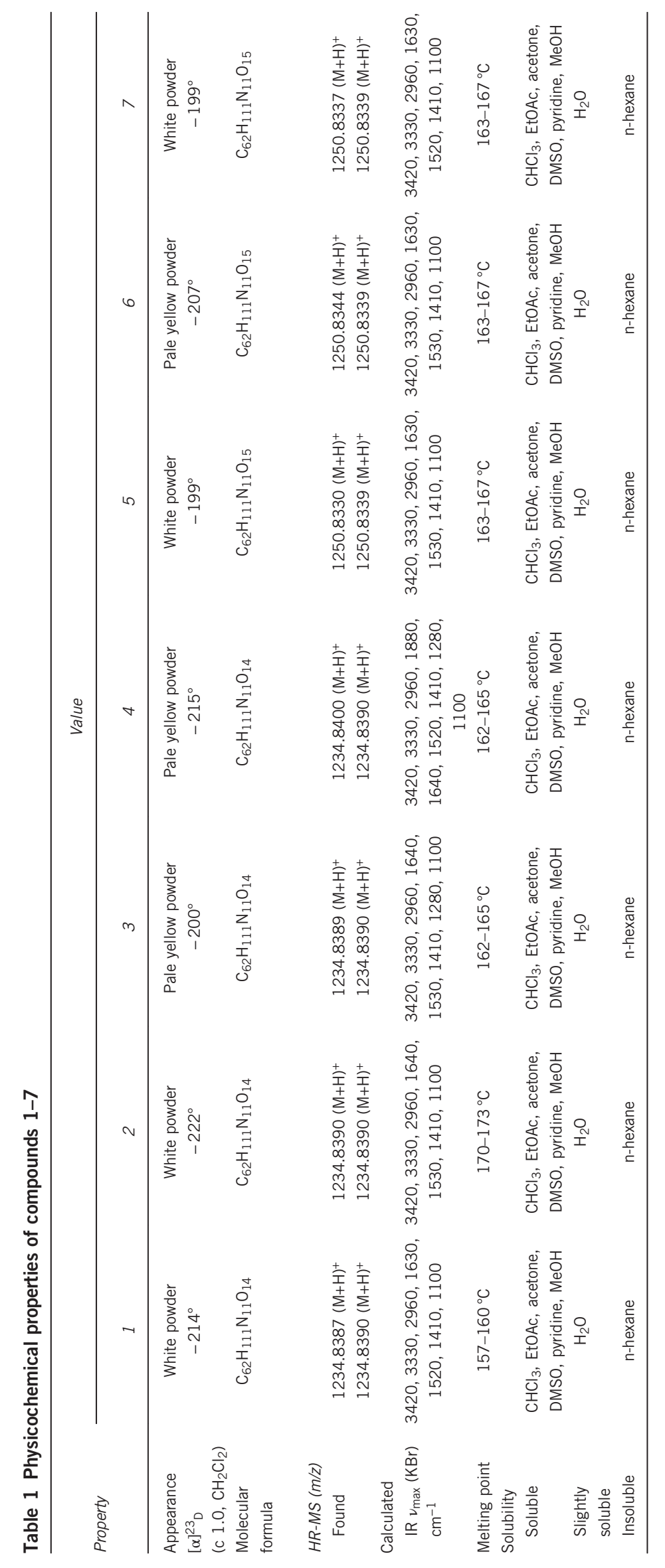


Table 2 NMR assignments of compounds 1-7 and FR901459

\begin{tabular}{|c|c|c|c|c|c|c|c|c|c|c|c|c|c|c|c|c|}
\hline \multirow[b]{2}{*}{ Position } & \multicolumn{2}{|c|}{ FR901459 } & \multicolumn{2}{|c|}{ (1) } & \multicolumn{2}{|c|}{ (2) } & \multicolumn{2}{|c|}{ (3) } & \multicolumn{2}{|c|}{ (4) } & \multicolumn{2}{|c|}{ (5) } & \multicolumn{2}{|c|}{ (6) } & \multicolumn{2}{|c|}{ (7) } \\
\hline & $\delta_{\mathrm{H}}$ & $\delta_{\mathrm{C}}$ & $\delta_{\mathrm{H}}$ & $\delta_{\mathrm{C}}$ & $\delta_{\mathrm{H}}$ & $\delta_{\mathrm{C}}$ & $\delta_{\mathrm{H}}$ & $\delta_{\mathrm{C}}$ & $\delta_{\mathrm{H}}$ & $\delta_{\mathrm{C}}$ & $\delta_{\mathrm{H}}$ & $\delta_{\mathrm{C}}$ & $\delta_{\mathrm{H}}$ & $\delta_{\mathrm{C}}$ & $\delta_{\mathrm{H}}$ & $\delta_{\mathrm{C}}$ \\
\hline \multicolumn{17}{|c|}{ 1-MeBmt } \\
\hline 1 & & 171.0 & & 170.96 & & 171.0 & & 171.0 & & 171.0 & & 170.95 & & 171.0 & & 171.0 \\
\hline 2 & 5.87 & 61.5 & 5.87 & 61.4 & 5.87 & 61.5 & 5.87 & 61.5 & 5.87 & 61.5 & 5.87 & 61.4 & 5.87 & 61.4 & 5.87 & 61.4 \\
\hline 3 & 4.70 & 75.2 & 4.75 & 75.0 & 4.69 & 75.2 & 4.69 & 75.2 & 4.70 & 75.2 & 4.75 & 74.9 & 4.75 & 75.0 & 4.74 & 75.0 \\
\hline 4 & 1.96 & 37.6 & 2.02 & 37.3 & 1.94 & 37.6 & 1.95 & 37.6 & 1.95 & 37.6 & 2.03 & 37.3 & 2.03 & 37.3 & 2.04 & 37.3 \\
\hline \multirow[t]{2}{*}{5} & 2.83 & 37.3 & 2.84 & 36.8 & 2.82 & 37.1 & 2.84 & 37.3 & 2.82 & 37.3 & 2.85 & 36.8 & 2.84 & 36.8 & 2.85 & 36.8 \\
\hline & 2.09 & & 2.29 & & 2.07 & & 2.08 & & 2.08 & & 2.31 & & 2.30 & & 2.31 & \\
\hline 6 & 5.56 & 129.8 & 5.95 & 128.7 & 5.55 & 129.8 & 5.56 & 129.8 & 5.56 & 129.8 & 5.95 & 128.7 & 5.94 & 128.7 & 5.95 & 128.7 \\
\hline 7 & 5.45 & 127.1 & 5.96 & 133.9 & 5.44 & 127.1 & 5.44 & 127.1 & 5.45 & 127.1 & 5.96 & 133.9 & 5.96 & 133.9 & 5.96 & 133.9 \\
\hline 8 & 1.59 & 18.1 & 4.35 & 63.1 & 1.59 & 18.1 & 1.59 & 18.1 & 1.59 & 18.1 & 4.35 & 63.1 & 4.35 & 63.1 & 4.34 & 63.1 \\
\hline 4-Me & 1.00 & 16.1 & 1.03 & 16.2 & 1.00 & 16.1 & 0.99 & 16.1 & 1.01 & 16.1 & 1.03 & 16.2 & 1.024 & 16.2 & 1.03 & 16.2 \\
\hline $\mathrm{N}-\mathrm{Me}$ & 4.23 & 34.1 & 4.23 & 34.1 & 4.23 & 34.1 & 4.23 & 34.2 & 4.23 & 34.1 & 4.24 & 34.1 & 4.23 & 34.1 & 4.23 & 34.1 \\
\hline $3-\mathrm{OH}$ & 7.81 & & 7.83 & & 7.81 & & 7.82 & & 7.81 & & 7.84 & & 7.84 & & 7.83 & \\
\hline $8-\mathrm{OH}$ & & & 6.06 & & & & & & & & 6.06 & & 6.06 & & 6.06 & \\
\hline \multicolumn{17}{|l|}{ 2-Thr } \\
\hline 1 & & 170.9 & & 170.91 & & 170.9 & & 170.9 & & 170.9 & & 170.91 & & 170.9 & & 170.96 \\
\hline 2 & 5.50 & 55.66 & 5.49 & 55.7 & 5.50 & 55.7 & 5.50 & 55.6 & 5.49 & 55.66 & 5.49 & 55.7 & 5.50 & 55.7 & 5.49 & 55.7 \\
\hline 3 & 4.59 & 69.6 & 4.58 & 69.6 & 4.59 & 69.6 & 4.59 & 69.6 & 4.59 & 69.6 & 4.58 & 69.6 & 4.58 & 69.6 & 4.58 & 69.6 \\
\hline 4 & 1.407 & 19.6 & 1.40 & 19.7 & 1.41 & 19.6 & 1.40 & 19.6 & 1.41 & 19.7 & 1.40 & 19.7 & 1.40 & 19.7 & 1.40 & 19.7 \\
\hline $\mathrm{NH}$ & 7.60 & & 7.61 & & 7.60 & & 7.60 & & 7.60 & & 7.61 & & 7.61 & & 7.61 & \\
\hline $3-\mathrm{OH}$ & 6.73 & & 6.74 & & 6.73 & & 6.74 & & 6.74 & & 6.75 & & 6.74 & & 6.75 & \\
\hline \multicolumn{17}{|l|}{ 3-Sar } \\
\hline 1 & & 169.5 & & 169.5 & & 169.5 & & 169.5 & & 169.5 & & 169.5 & & 169.5 & & 169.5 \\
\hline 2 & 4.95 & 49.0 & 4.95 & 49.0 & 4.94 & 49.0 & 4.94 & 49.0 & 4.94 & 49.0 & 4.94 & 49.0 & 4.95 & 49.0 & 4.94 & 49.0 \\
\hline & 3.39 & & 3.38 & & 3.39 & & 3.39 & & 3.39 & & 3.39 & & 3.39 & & 3.38 & \\
\hline $\mathrm{N}-\mathrm{Me}$ & 3.47 & 39.1 & 3.47 & 39.1 & 3.47 & 39.1 & 3.47 & 39.1 & 3.47 & 39.1 & 3.47 & 39.1 & 3.47 & 39.1 & 3.47 & 39.1 \\
\hline 4-MeLeu & & & & & & & & & & & & & & & & \\
\hline 1 & & 169.0 & & 169.0 & & 169.0 & & 169.0 & & 169.0 & & 169.0 & & 169.0 & & 169.0 \\
\hline 2 & 5.10 & 59.3 & 5.10 & 59.3 & 5.10 & 59.3 & 5.10 & 59.3 & 5.10 & 59.3 & 5.10 & 59.3 & 5.11 & 59.3 & 5.10 & 59.3 \\
\hline 3 & 2.03 & 38.2 & 2.04 & 38.2 & 2.03 & 38.2 & 2.01 & 38.2 & 2.03 & 38.2 & 2.03 & 38.2 & 2.03 & 38.2 & 2.03 & 38.2 \\
\hline & 1.56 & & 1.53 & & 1.53 & & 1.53 & & 1.53 & & 1.54 & & 1.53 & & 1.54 & \\
\hline 4 & 1.58 & 24.9 & 1.58 & 24.9 & 1.56 & 24.9 & 1.57 & 24.9 & 1.56 & 24.9 & 1.57 & 24.9 & 1.57 & 24.9 & 1.58 & 24.9 \\
\hline 5 & 0.926 & 23.2 & 0.93 & 23.2 & 0.93 & 23.2 & 0.93 & 23.2 & 0.93 & 23.2 & 0.93 & 23.20 & 0.93 & 23.20 & 0.93 & 23.21 \\
\hline 6 & 0.926 & 22.8 & 0.92 & 22.8 & 0.92 & 22.8 & 0.92 & 22.8 & 0.92 & 22.8 & 0.92 & 22.8 & 0.92 & 22.8 & 0.92 & 22.8 \\
\hline $\mathrm{N}-\mathrm{Me}$ & 2.98 & 28.8 & 2.98 & 28.8 & 2.98 & 28.8 & 2.98 & 28.8 & 2.98 & 28.8 & 2.98 & 28.8 & 2.98 & 28.8 & 2.98 & 28.8 \\
\hline 5-Leu & & & & & & & & & & & & & & & & \\
\hline 1 & & 173.4 & & 173.4 & & 173.4 & & 173.4 & & 173.4 & & 173.4 & & 173.4 & & 173.4 \\
\hline 2 & 5.40 & 48.1 & 5.40 & 48.1 & 5.40 & 48.1 & 5.40 & 48.1 & 5.40 & 48.1 & 5.41 & 48.1 & 5.40 & 48.1 & 5.40 & 48.1 \\
\hline 3 & 1.70 & 41.2 & 1.70 & 41.2 & 1.70 & 41.2 & 1.69 & 41.3 & 1.70 & 41.2 & 1.71 & 41.2 & 1.70 & 41.2 & 1.70 & 41.2 \\
\hline & 1.45 & & 1.43 & & 1.43 & & 1.43 & & 1.43 & & 1.44 & & 1.43 & & 1.43 & \\
\hline 4 & 1.63 & 24.8 & 1.63 & 24.8 & 1.62 & 24.8 & 1.62 & 24.8 & 1.62 & 24.8 & 1.62 & 24.8 & 1.62 & 24.8 & 1.62 & 24.8 \\
\hline 5 & 0.932 & 20.9 & 0.92 & 20.9 & 0.92 & 20.9 & 0.93 & 20.9 & 0.92 & 20.9 & 0.93 & 20.9 & 0.92 & 20.9 & 0.93 & 20.9 \\
\hline 6 & 0.57 & 23.20 & 0.57 & 23.2 & 0.57 & 23.2 & 0.56 & 23.2 & 0.57 & 23.21 & 0.57 & 23.20 & 0.57 & 23.20 & 0.57 & 23.21 \\
\hline $\mathrm{NH}$ & 8.43 & & 8.42 & & 8.43 & & 8.43 & & 8.43 & & 8.42 & & 8.42 & & 8.42 & \\
\hline 6-MeLeu & & & & & & & & & & & & & & & & \\
\hline 1 & & 173.0 & & 172.95 & & 173.0 & & 172.97 & & 173.0 & & 172.95 & & 172.95 & & 172.94 \\
\hline 2 & 5.95 & 54.6 & 5.93 & 54.6 & 5.95 & 54.6 & 5.95 & 54.6 & 5.93 & 54.6 & 5.94 & 54.6 & 5.94 & 54.6 & 5.93 & 54.6 \\
\hline 3 & 2.40 & 38.5 & 2.38 & 38.5 & 2.41 & 38.5 & 2.45 & 38.6 & 2.40 & 38.5 & 2.43 & 38.6 & 2.41 & 38.5 & 2.39 & 38.5 \\
\hline & 1.92 & & 1.91 & & 1.91 & & 1.91 & & 1.89 & & 1.91 & & 1.91 & & 1.89 & \\
\hline 4 & 1.76 & 25.9 & 1.75 & 25.9 & 1.75 & 25.9 & 1.75 & 25.9 & 1.75 & 25.9 & 1.75 & 25.9 & 1.75 & 25.9 & 1.73 & 25.9 \\
\hline 5 & 1.08 & 23.20 & 1.06 & 23.2 & 1.07 & 23.2 & 1.09 & 23.2 & 1.03 & 23.23 & 1.09 & 23.26 & 1.08 & 23.24 & 1.03 & 23.21 \\
\hline 6 & 1.02 & 23.27 & 1.02 & 23.3 & 1.02 & 23.3 & 1.02 & 23.3 & 0.97 & 23.25 & 1.02 & 23.26 & 1.017 & 23.28 & 0.97 & 23.24 \\
\hline $\mathrm{N}-\mathrm{Me}$ & 3.37 & 31.0 & 3.36 & 31.0 & 3.37 & 31.0 & 3.37 & 31.0 & 3.37 & 31.0 & 3.37 & 31.0 & 3.36 & 31.0 & 3.37 & 31.0 \\
\hline
\end{tabular}


Table 2 (Continued)

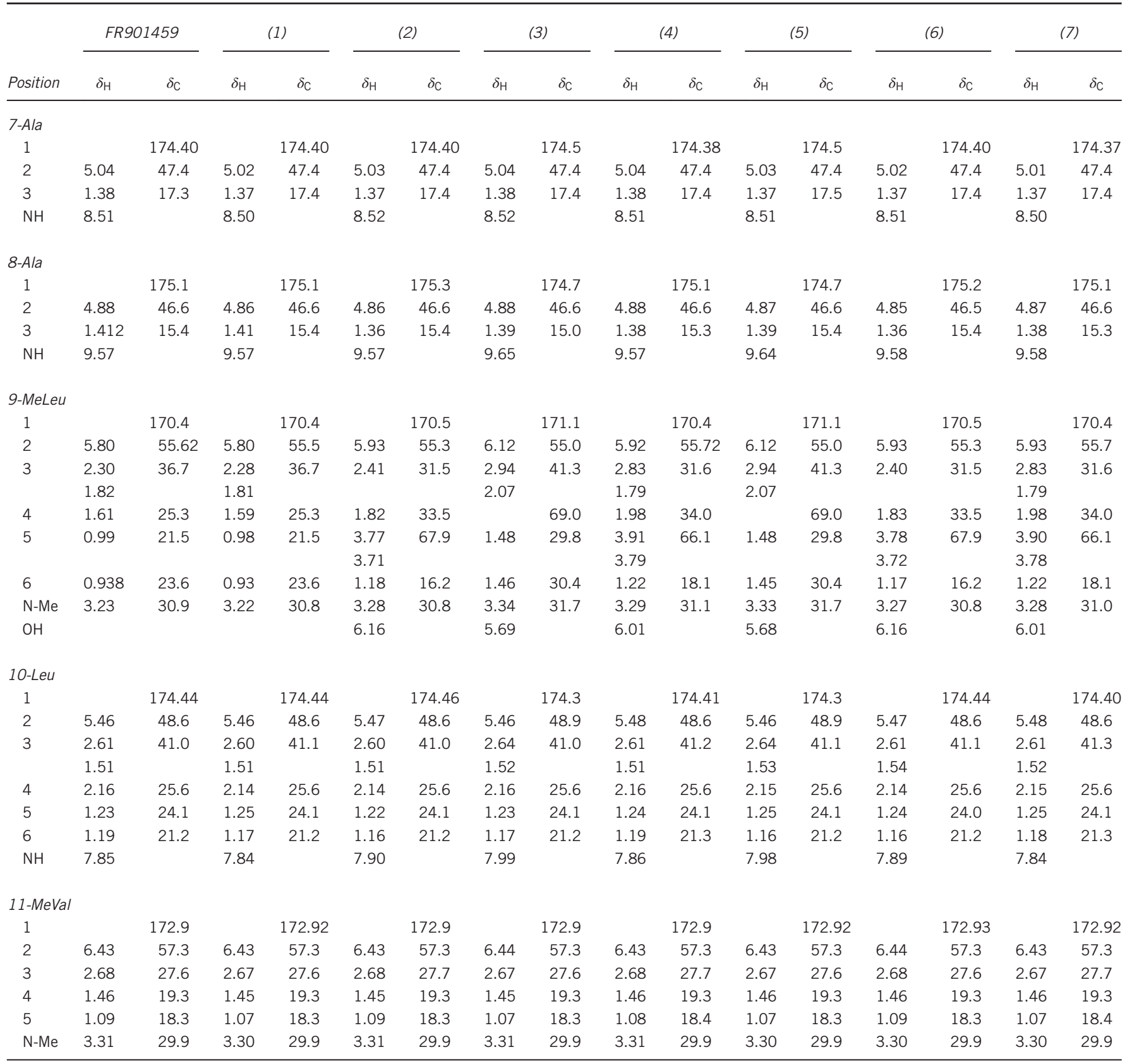

Abbreviations: Bmt, (4R)-4-[(E)-2-butenyl]-4-methyl-L-threonine; Me, methyl; Sar, sarcosine.

NMR spectra, ${ }^{1} \mathrm{H}(500 \mathrm{MHz})$ and ${ }^{13} \mathrm{C}(125 \mathrm{MHz})$, were recorded on a Bruker DRX 500 spectrometer equipped with a cryoprobe.

7887 and members of the genus Lentzea were between $97.1 \%$ (L. californiensis) and $99.2 \%$ (L. violacea). These species comprised a single cluster on the phylogenetic tree (Figure 5).

Based on the morphological and chemical characteristics of strain 7887 , and the results of the phylogenetic analysis, we concluded that strain 7887 belonged to the genus Lentzea. ${ }^{15-17}$ Therefore, this strain was designated as Lentzea sp. 7887.

\section{Evaluation of members of the genus Lentzea for bioconversion capability}

To evaluate the bioconversion activity of strain 7887 , the bioconversion products generated by six other strains of Lentzea were analyzed as described above for the reaction products of strain 7887. HPLC analysis revealed that all of the examined strains converted FR901459 to derivatives 1-7 (Table 5). Compound 3 was the major conversion compound produced by four of the Lentzea strains. In contrast, strain 7887 only produced relatively low levels of compound 3 , but formed compounds $\mathbf{2}$ and $\mathbf{6}$ at markedly higher rates than all of the other strains. Taken together, these results suggested that several members of the genus Lentzea are suitable for metabolizing FR901459, and that strain 7887 has a unique profile for the bioconversion of FR901459 to potentially useful compounds.

\section{DISCUSSION}

The CsA analog FR901459 is a useful chemical template for the development and design of new classes of anti-HCV drugs; however, 


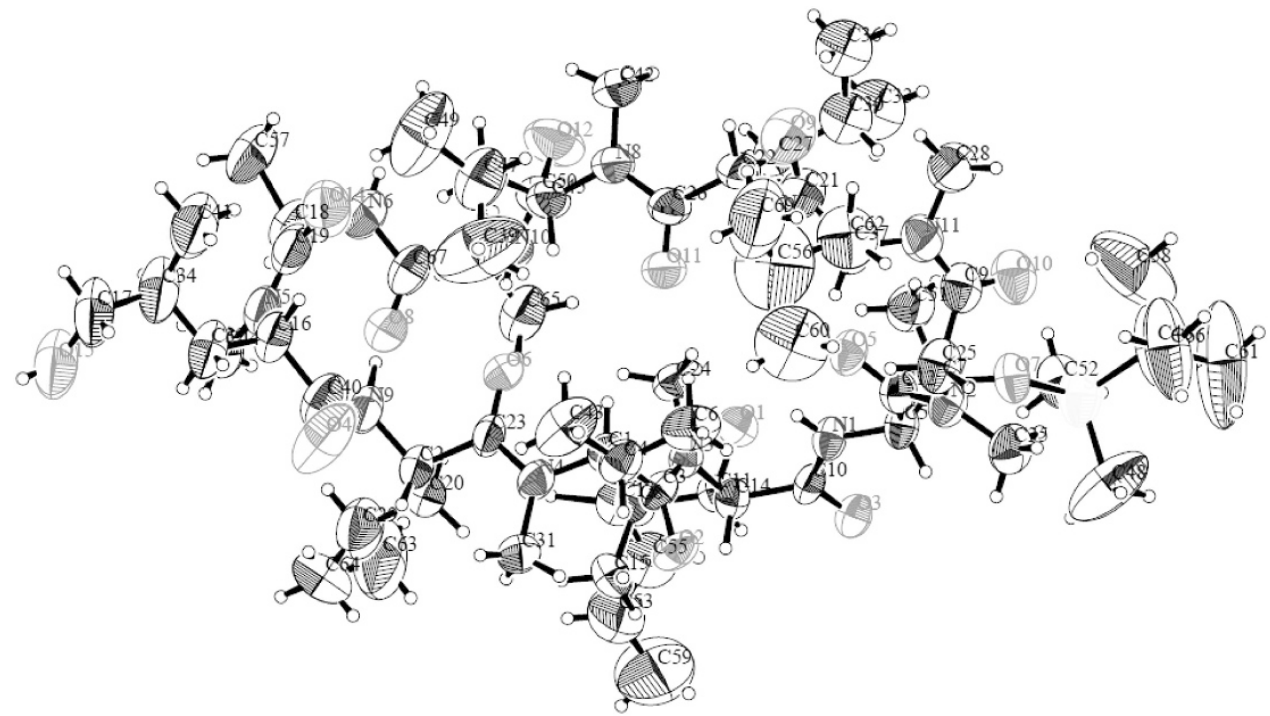

Figure 3 ORTEP drawing of FR310363. A full color version of this figure is available at The Journal of Antibiotics journal online.

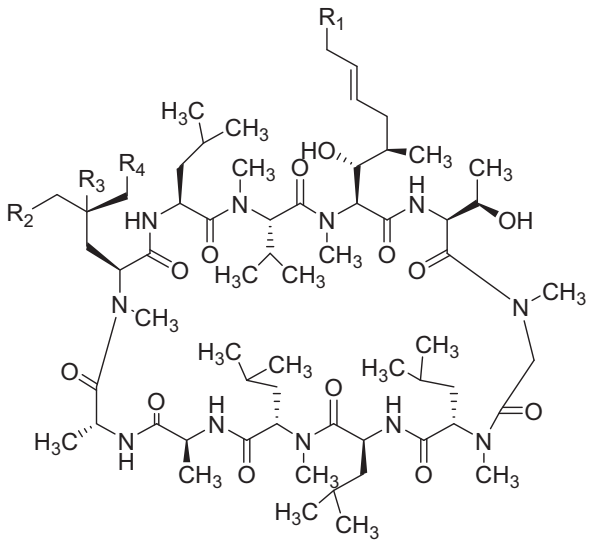

\begin{tabular}{c|ccccccc}
\hline & $\mathbf{1}$ & $\mathbf{2}$ & $\mathbf{3}$ & $\mathbf{4}$ & $\mathbf{5}$ & $\mathbf{6}$ & $\mathbf{7}$ \\
\hline \hline $\mathrm{R}_{1}$ & $\mathrm{OH}$ & $\mathrm{H}$ & $\mathrm{H}$ & $\mathrm{H}$ & $\mathrm{OH}$ & $\mathrm{OH}$ & $\mathrm{OH}$ \\
\hline $\mathrm{R}_{2}$ & $\mathrm{H}$ & $\mathrm{OH}$ & $\mathrm{H}$ & $\mathrm{H}$ & $\mathrm{H}$ & $\mathrm{OH}$ & $\mathrm{H}$ \\
\hline $\mathrm{R}_{3}$ & $\mathrm{H}$ & $\mathrm{H}$ & $\mathrm{OH}$ & $\mathrm{H}$ & $\mathrm{OH}$ & $\mathrm{H}$ & $\mathrm{H}$ \\
\hline $\mathrm{R}_{4}$ & $\mathrm{H}$ & $\mathrm{H}$ & $\mathrm{H}$ & $\mathrm{OH}$ & $\mathrm{H}$ & $\mathrm{H}$ & $\mathrm{OH}$ \\
\hline
\end{tabular}

Figure 4 Structures of bioconversion products (compounds 1-7) derived from FR901459.

Table 3 Cultural characteristics of strain 7887

\begin{tabular}{|c|c|c|c|c|}
\hline \multirow[b]{2}{*}{ Medium } & \multicolumn{4}{|c|}{ Characteristic } \\
\hline & Growth & Aerial mycelia & Reverse side color & Soluble pigment \\
\hline Oatmeal agar (ISP-3) & Moderate & Thin, white & Orange red (8A8) & Orange \\
\hline Inorganic salts-starch agar (ISP-4) & Good & Scant, white & Brownish red $(9 \mathrm{C} 7)$ & Light brown \\
\hline Glycerol-asparagine agar (ISP-5) & Good & Moderate, white & Brownish orange (7C7) & Brown \\
\hline Benett's agar & Good & Thin, white & Orange red (8B8) & Brown \\
\hline Czapek agar & Moderate & Moderate, white & Orange (6A6) & Light orange \\
\hline Yeast-starch agar & Moderate & Moderate, white & Orange red (8B8) & Light brown \\
\hline Glucose-asparagine agar & Moderate & Moderate, yellowish white (4A2) & Pale orange (5A3) & Light brown \\
\hline Sucrose-nitrate agar & Moderate & Thin, yellowish white (4A2) & Orange (5A6) & Light brown \\
\hline
\end{tabular}

this compound is resistant to chemical modification. Because bioconversion has the potential to efficiently hydroxylate chemically resistant compounds; here, we screened for actinomycete strains capable of bioconverting FR901459, and included actinomycete strains that reportedly catalyze the conversion other compounds, such as AS1387392. ${ }^{18}$ We identified a novel isolate, strain 7887, which was capable of converting FR901459 to several bioconversion products (1-7). Isolation and structural elucidation of the derivatization 
Table 4 Physiological characteristics of strain 7887

\begin{tabular}{|c|c|}
\hline Parameter & Characteristic \\
\hline Temperature range for growth $\left({ }^{\circ} \mathrm{C}\right)$ & $10.5-35.2$ \\
\hline Optimum temperature for growth $\left({ }^{\circ} \mathrm{C}\right)$ & 31.0 \\
\hline Production of melanoid pigments & - \\
\hline Production of soluble pigments & + \\
\hline Hydrolysis of gelatin & + \\
\hline \multicolumn{2}{|l|}{ Carbon utilization } \\
\hline L-Arabinose & + \\
\hline D-Xylose & + \\
\hline D-Glucose & + \\
\hline D-Fructose & + \\
\hline Sucrose & + \\
\hline L-Rhamnose & - \\
\hline Raffinose & - \\
\hline Inositol & + \\
\hline D-Mannitol & + \\
\hline Adonitol & - \\
\hline Cellobiose & + \\
\hline Dextran & - \\
\hline Dextrin & + \\
\hline Dulcitol & - \\
\hline D-Galactose & + \\
\hline Glycerol & + \\
\hline Inulin & - \\
\hline Lactose & - \\
\hline D-Mannose & + \\
\hline Maltose & + \\
\hline D-Melezitose & - \\
\hline D-Melibiose & + \\
\hline Sorbitol & - \\
\hline Soluble starch & + \\
\hline L-Sorbose & - \\
\hline Trehalose & + \\
\hline Xylitol & - \\
\hline Sodium acetate $(0.1 \% \mathrm{v} / \mathrm{v})$ & \pm \\
\hline Tri-sodium citrate $(0.1 \% \mathrm{v} / \mathrm{v})$ & \pm \\
\hline Malonic acid disodium salt $(0.1 \% \mathrm{v} / \mathrm{v})$ & \pm \\
\hline Propionic acid sodium salt $(0.1 \% \mathrm{v} / \mathrm{v})$ & \pm \\
\hline Pyruvic acid sodium salt $(0.1 \%$ v/v) & \pm \\
\hline
\end{tabular}

+ , utilized; \pm , weakly utilized; -, not utilized.

products revealed that this strain was able to efficiently hydroxylate two positions of FR901459: the methyl-Bmt group at position 1, generating compound $\mathbf{1}$; the $\mathrm{N}$-methyl-Leu group at position 9, generating compounds $2-4$; and both positions 1 and 9, generating compounds 5-7. These compounds might serve as useful chemical templates that can be further modified to produce novel antiHCV drugs.

Compound 2 was generated from FR901459 by strain 7887 at a reasonably high rate. It was previously reported that compounds chemically derived from compound 2 had potent anti-HCV effects, but reduced immunosuppressive activity, and that further derivatization of the three-position generated compounds with powerful antiHCV activity and excellent pharmacokinetic profiles. ${ }^{19}$ Thus, strain 7887 may be useful for the large-scale production of compound $\mathbf{2}$ as a template for the development of highly effective and safe anti-HCV agents.

Morphological and chemical characterization and phylogenetic analysis of $16 \mathrm{~S}$ rDNA indicated that strain 7887 belongs to the genus

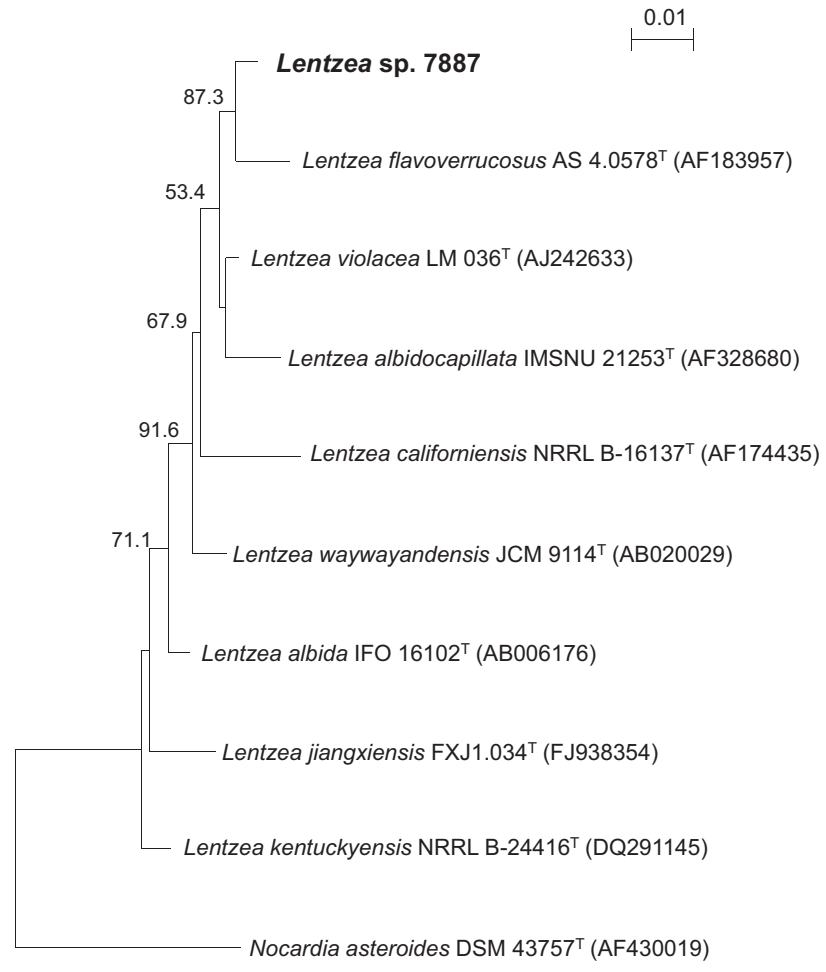

Figure 5 Phylogenetic tree based on near full-length 16S rDNA sequences ( 1400 nucleotides) showing the position of strain 7887 . The numbers on the branches are confidence limits (expressed as percentages) estimated from a bootstrap analysis with 1000 replicates (limits $>50 \%$ are indicated). Scale bar, 0.01 substitutions per nucleotide position.

Table 5 Bioconversion capabilities of members of the genus Lentzea

\begin{tabular}{lcccccccc}
\hline & \multicolumn{7}{c}{ Conversion rate $^{\mathrm{a}}(\%)$} \\
\cline { 2 - 9 } Strain & 1 & 2 & 3 & 4 & 5 & 6 & 7 \\
\hline Lentzea sp. 7887 $^{n}$ & 6.2 & 28.0 & 5.6 & 2.6 & 2.6 & 17.0 & 4.6 \\
Lentzea violacea JCM 10975 $^{\top}$ & 1.0 & 15.0 & 20.2 & 4.6 & 5.2 & 3.0 & 1.6 \\
Lentzea albidocapillata JCM 9732 $^{\top}$ & 1.0 & 13.0 & 27.4 & 3.2 & 9.8 & 3.4 & 2.2 \\
Lentzea flaviverrucosa JCM 11373 $^{\top}$ & 1.4 & 3.2 & 5.0 & 0.8 & 0 & 0 & 0 \\
Lentzea californiensis JCM 11305 $^{\top}$ & 2.2 & 16.8 & 20.8 & 2.8 & 7.2 & 4.6 & 2 \\
Lentzea waywayandensis JCM 9114 $^{\top}$ & 3.6 & 9.0 & 28.4 & 1.4 & 11.4 & 2.0 & 0.8 \\
Lentzea albida JCM 10670 $^{\top}$ & 5.6 & 10.2 & 5.2 & 2.4 & 0.8 & 0.8 & 0
\end{tabular}

Abbreviation: JCM, Japan Collection of Microorganisms.

${ }^{a}$ Conversion rate: (amount of the derivative produced/amount of FR901459 added) $\times 100$.

Lentzea. ${ }^{15-17}$ Strain 7887 could be distinguished from the most closely related strain, L. violacea LM036 ${ }^{\mathrm{T}}$ based on phenotypic characteristics, including the temperature range for growth, substrate mycelium color and utilization of L-rhamnose, adonitol and lactose (data from Lee et al.). ${ }^{20}$ We therefore suggest that strain 7887 belongs to a new species of the genus Lentzea, but further study will be needed.

Nearly, all of the Lentzea strains examined in the present study were able to convert FR901459 to derivative compounds. In a preliminary study, several members of the closely related genera Saccharothirix, Actinokinespora, Lechevalieria and Actinosynnema only poorly metabolized FR901459, as demonstrated by the low amount of compound 1 produced by these strains. These results indicate that Lentzea strains have superior FR901459 bioconversion activity. However, within the 
genus Lentzea, widely varying bioconversion rates and products were observed, at least under the present culture conditions. In view of the high productivity of compound 2 , strain 7887 is considered to be the most promising strain among those examined in the present study for the synthesis of bioactive compounds from FR901459.

In conclusion, we successfully identified a new strain Lentzea sp. 7887 that is capable of bioconverting the CsA derivative FR901459 into a chemically accessible template that is amenable for further modification. We anticipate that the findings from this study will lead to the targeted development of more effective and safer anti-HCV drugs.

\section{METHODS}

\section{Materials}

FR901459 was isolated from the fermentation broth of S. chartarum No. 19392, as previously reported. ${ }^{13}$ The actinomycete Lentzea sp. 7887 was isolated from a soil sample collected in Ibaraki Prefecture, Japan. All other Lentzea strains used in this study were purchased from the Japan Collection of Microorganisms.

\section{Culture media}

Medium A consisted of 1\% corn flour, 6\% MS\#3600 (Nihon Shokuhin Kako Co., Ltd, Tokyo, Japan), 1.2\% Pharmamedia (Archer Daniels Midland Company, Lubbock, IL, USA), $0.8 \%$ dried yeast, $0.3 \% \quad \mathrm{KH}_{2} \mathrm{PO}_{4}, 0.3 \%$ $\mathrm{MgSO}_{4} \cdot 7 \mathrm{H}_{2} \mathrm{O}$ and $0.02 \% \mathrm{FeSO}_{4} \cdot 7 \mathrm{H}_{2} \mathrm{O}(\mathrm{pH}$ 6.5). Medium B consisted of $0.5 \%$ glucose, $0.5 \%$ sucrose, $0.5 \%$ oatmeal, $0.2 \%$ yeast extract, $0.5 \%$ peptone, $0.5 \%$ peanut powder, $0.01 \%$ humic acid, $0.1 \%$ Tween 80 and $0.5 \% \mathrm{CaCO}_{3}$ ( $\mathrm{pH} 7.0)$.

\section{Bioconversion of FR901459 using Lentzea sp. 7887}

Aqueous medium A $(60 \mathrm{ml})$ in a $225-\mathrm{ml}$ Erlenmeyer flask was sterilized at $121^{\circ} \mathrm{C}$ for $30 \mathrm{~min}$, cooled and then inoculated with a loopful of a slant culture of Lentzea sp. 7887. After incubation at $30^{\circ} \mathrm{C}$ with shaking at 250 r.p.m. for 3 days, $3.2 \mathrm{ml}$ of the seed culture was transferred to a 500-ml Erlenmeyer flask containing $160 \mathrm{ml}$ medium B. The flasks were then further incubated at $30^{\circ} \mathrm{C}$ with shaking at 250 r.p.m. for 3 days, and 3.2 and 41 of the second seed culture was then inoculated into 200- and 300-1 jar fermentors, respectively, containing 160 and 2001 of production medium A containing $0.1 \%$ Adekanol (Adeka Co., Ltd, Tokyo, Japan) and silicone $0.1 \%$ (Shin-Etsu Chemical Co., Ltd, Tokyo, Japan), respectively. Fermentation was performed at $30^{\circ} \mathrm{C}$ with stirring at 200 r.p.m. for 3 days. After the culture period, a solution of FR901459 (180 g) in methanol (7.2l) was added to 3601 of the cultured broth. The reaction mixture was then incubated at $30^{\circ} \mathrm{C}$ with constant stirring for $13 \mathrm{~h}$ and the reaction products were analyzed by HPLC, as described below.

\section{Assay for anti-HCV activity}

Inhibitory activity against replication of the HCV replicon was evaluated by determining the amount of replicon RNA using real-time quantitative reverse transcription (RT)-PCR. ${ }^{21,22}$ HCV replicon cells (\#50-1, a kind gift from Dr K Shimotohno, Kyoto University) were cultured in Dulbecco's Modified Eagle medium (DMEM) containing 5\% fetal bovine serum and $300 \mu \mathrm{g} \mathrm{ml}^{-1} \mathrm{G} 418$ at $37^{\circ} \mathrm{C}$ in $5 \% \mathrm{CO}_{2} \cdot{ }^{23}$ After incubation for 2 days with the test compounds, total RNA was isolated from cells using the RNeasy kit (Qiagen Inc., Tokyo, Japan) according to the manufacturer's protocol. The quantitative RT-PCR was performed using the TaqMan Ez RT-PCR Core Reagent (Applied Biosystems Inc., Foster City, CA, USA). The sequences of the primer set for amplifying part of the HCV genome were as follows:

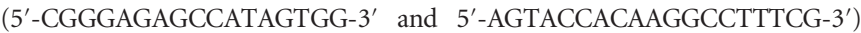
and the complementary probe (5'-FAM-CTGCGGAACCGTGAGTACAC TAMRA-3'; Takara Bio Inc., Shiga, Japan). The quantitative RT-PCR was performed using the ABI PRISM 7900HT sequence detection system (Applied Biosystems Inc.). The standard curve for this assay was calculated using a series of 10-fold dilutions of synthetic HCV RNA (a kind gift from Dr M Kohara, Tokyo Metropolitan Institute of Medical Science). The copy number was calibrated to the levels of the housekeeping gene encoding glyceraldehyde-3- phosphate dehydrogenase. $\mathrm{IC}_{50}$ values of the compounds were determined from the 50\% replicon RNA levels corrected by the intrinsic control glyceraldehyde-3-phosphate dehydrogenase values.

\section{Taxonomic characterization of Lentzea sp. 7887}

Strain 7887 was taxonomically characterized using the methods and media described by Hamada. ${ }^{24}$ All observations were made after 14-day cultivation at $30^{\circ} \mathrm{C}$.

Morphological observations were made using an optical microscope and a scanning electron microscope (Hitachi S-2600N, Hitachi High-Technologies Corp., Tokyo, Japan) on cultures grown on $1 / 5$ yeast extract-starch agar, which was composed of $0.4 \mathrm{~g}$ yeast extract (Daigo Eiyo, Osaka, Japan), $2 \mathrm{~g}$ soluble starch and $16 \mathrm{~g}$ agar in 11 tap water ( $\mathrm{pH}$ was adjusted to 7.2 with $1 \mathrm{~N} \mathrm{NaOH}$ before sterilization). The temperature growth range of strain 7887 was also determined using 1/5 yeast extract-starch agar. Carbon utilization was evaluated on Pridham and Gottlieb's medium. ${ }^{25}$ All color names used in this study were taken from the Methuen Handbook of Colour. ${ }^{26}$ The chemotaxonomic characterization of strain 7887 was performed by the procedure of Suzuki et al. ${ }^{27}$ Cell preparation and diaminopimelic acid isomer detection were conducted following the procedures developed by Becker et al. ${ }^{28}$

\section{$16 S$ rDNA sequencing and phylogenetic analysis}

The $16 \mathrm{~S} \mathrm{rDNA}$ sequence of strain 7887 was determined using the method developed by Nakagawa and Kawasaki, ${ }^{29}$ and was compared with $16 \mathrm{~S}$ rDNA sequences of type strains obtained from the DNA Data Bank of Japan (http:// www.ddbj.nig.ac.jp) by BLAST search. A phylogenetic tree was then constructed using the alignment program ClustalW. ${ }^{30}$ The DDBJ/GenBank/EMBL accession number for $16 \mathrm{~S}$ rDNA sequence of strain 7887 is AB986227.

\section{Evaluation of bioconversion activity of Lentzea strains}

Each strain was grown in tubes $(17 \mathrm{~mm} \times 100 \mathrm{~mm})$ containing $1.5 \mathrm{ml}$ medium A. The culture tubes were inoculated with a loopful of a slant culture and then incubated at $30^{\circ} \mathrm{C}$ with shaking at 250 r.p.m. for 3 days. After incubation, $0.5 \mathrm{ml}$ of culture broth was transferred to a tube containing $0.5 \mathrm{mg} \mathrm{ml}^{-1}$ FR901459, and the reaction mixtures were further incubated at $30^{\circ} \mathrm{C}$ for $9 \mathrm{~h}$ with shaking at 250 r.p.m. Extracts were prepared by mixing the reaction mixture with equal volumes of acetone followed by centrifugation of the extracts $(3000 \mathrm{~g}, 10 \mathrm{~min})$. The resulting supernatants were analyzed by HPLC (Agilent HP1100; Agilent Technologies, Inc., Santa Clara, CA, USA) using an ODS column (Mightysil RP-18 GP, $100 \mathrm{~mm} \times 2.0 \mathrm{~mm}$ I.D.; Kanto Chemical Co., Inc.). The elution solvents were water containing $0.1 \%$ trifluoroacetic acid (solvent A) and acetonitrile containing $0.1 \%$ trifluoroacetic acid (solvent B). A 15 -min linear gradient from 45 to $100 \%$ solvent B was used with a 2-min hold and a flow rate of $0.4 \mathrm{ml} \mathrm{min}{ }^{-1}$. The detection wavelength was set at $210 \mathrm{~nm}$ and the column was heated at $50^{\circ} \mathrm{C}$. Under these conditions, FR901459 was eluted at a retention time of $16.9 \mathrm{~min}$ and compounds $1-7$ were eluted at retention times of $10.9,8.5,10.2,9.4,5.0,4.0$ and $4.2 \mathrm{~min}$, respectively.

\section{CONFLICT OF INTEREST}

The authors declare no conflict of interest.

\section{ACKNOWLEDGEMENTS}

HCV replicon cells (\#50-1) were kindly provided by Dr K Shimotohno (Kyoto University). We would like to thank Dr M Kohara (Tokyo Metropolitan Institute of Medical Science) for advising about quantification of the HCV genome. We also thank Dr Shigehiro Takase for suggestion and technical assistance with structure elucidation and Ms Mayumi Sodeyama for technical assistance with isolation of actinomycetes.

1 Hosobuchi, M., Kurosawa, K. \& Yoshikawa, H. Application of computer to monitoring and control of fermentation process: Microbial conversion of ML-236B Na to pravastatin. Biotechnol. Bioeng. 42, 815-820 (1993). 
2 Ueda, S. et al. Strain selection and scale-up fermentation for FR901379 acylase production by Streptomyces sp. no. 6907. J. Biosci. Bioeng. 112 409-414 (2011).

3 Sasaki, J. et al. Transformation of vitamin $D_{3}$ to $1 \alpha, 25$-dihydroxyvitamine $D_{3}$ via 25-hydroxyvitamine $\mathrm{D}_{3}$ using Amycolata sp. strains. Appl. Microbiol. Biotechnol. 38 152-157 (1992).

4 Schulman, M., Doherty, P., Zink, D. \& Arison, B. Microbial conversion of avermectins by Saccharopolyspora erthraea hydroxylation at C-27. J. Antibiot. 47, 372-375 (1994).

5 Nakagawa, K. \& Torikata, A. Microbial conversion of milbemycins: 30-oxidation of milbemycin A4 and related compounds by Amycolatata autotrophica and amycolatopsis mediterranei. J. Antibiot. 43, 1321-1328 (1990).

6 Hall, R. M. et al. The production of novel sordarin analogues by transformation J. Antibiot. 54, 948-957 (2001).

7 Kobayashi, M. et al. FR177391, a new anti-hyperlipidemic agent from Serratia. J. Antibiot. 58, 648-653 (2005).

$8 \mathrm{Ho}$, S. et al. The mechanism of action of cyclosporin A and FK506. Clin. Immunol. Immunopathol. 80, S40-S45 (1996).

9 Watashi, K., Hijikata, M., Hosaka, M., Yamaji, M. \& Shimotohno, K. Cyclosporin A suppresses replication of hepatitis $\mathrm{C}$ virus genome in cultured hepatocytes. Hepatology 38, 1282-1288 (2003).

10 Nakagawa, M. et al. Suppression of hepatitis C virus replication by cyclosporin a is mediated by blockade of cyclophilins. Gastroenterology 129, 1031-1041 (2005).

11 Inoue, K. et al. Combined interferon alpha2b and cyclosporin A in the treatment of chronic hepatitis C: controlled trial. J. Gastroenterol. 38, 567-572 (2003).

12 Membreno, E. E., Espinales, J. C. \& Lawitz, E. J. Cyclophilin inhibitors for hepatitis C therapy. Clin. Liver Dis. 17, 129-139 (2013).

13 Sakamoto, K. et al. FR901459, a novel immunosuppressant isolated from Stachybotrys chartarum No. 19392. J. Antibiot. 46, 1788-1798 (1993).

14 Muramatsu, Y. et al. Neuroprotective efficacy of FR901459, a novel derivative of cyclosporin $\mathrm{A}$, in in vitro mitochondrial damage and in vivo transient cerebral ischemia models. Brain Res. 1149, 181-190 (2007).

15 Yassin, A. F. et al. Lentzea gen. nov., a new genus of the order Actinomycetales. Int. J. Syst. Bacteriol. 45, 357-363 (1995).

16 Labeda, D. P., Hatano, K., Kroppenstedt, R. M. \& Tamura, T. Revival of the genus Lentzea and proposal for Lechevalieria gen. nov. Int. J. Syst. Evol. Microbiol. 51 1045-1050 (2001).
17 Xie, Q. et al. Description of Lentzea flaviverrucosa sp. nov. and transfer of the type strain of Saccharothrix aerocolonigenes subsp. staurosporea to Lentzea albida. Int. J. Syst. Evol. Microbiol. 52, 1815-1820 (2002).

18 Sasamura, S. et al. Bioconversion of AS1387392: screening and characterization of actinomycetes that convert AS1387392 to AS1429736. J. Antibiot. 63 637-642 (2010).

19 Yamanaka, T. et al. Synthetic studies on anti-HCV agents via chemical transformation of cyclosporin analogues (2). The 31st Medicinal Chemistry Symp 1P-41 (The Pharmaceutical Society of Japan, Hiroshima, Japan, 2013).

20 Lee, S. D. et al. Saccharothrix violacea sp. nov., isolated from a gold mine cave, and Saccharothrix albidocapillata comb. nov. Int. J. Syst. Evol. Microbiol. 50, 1315-1323 (2000).

21 Takeuchi, T. et al. Real-time detection system for quantification of hepatitis C virus genome. Gastroenterology 116, 636-642 (1999).

22 Lohmann, V. et al. Replication of subgenomic hepatitis C virus RNAs in a hepatoma cell line. Science 285, 110-113 (1999).

23 Kishine, $\mathrm{H}$. et al. Subgenomic replicon derived from a cell line infected with the hepatitis C virus. Biochem. Biophys. Res. Commun. 293, 993-999 (2002).

24 Hamada, M. Identification Manual of Actinomycetes (in Japanese), (ed.The Society for Actinomycetes Japan) 37-47 (Business Center for Academic Societies Japan, Tokyo, Japan, 2001).

25 Pridham, T. G. \& Gottlieb, D. The utilization of carbon compounds by some Actinomycetales as an acid for species determination. J. Bacteriol. 56 107-114 (1948).

26 Kornerup, A. \& Wanscher, J. H. Methuen Handbook of Colour (Methuen, London, 1978).

27 Suzuki, K. \& Kudo, T. Identification Manual of Actinomycetes. (in Japanese), (ed. The Society for Actinomycetes Japan) 49-82 (Business Center for Academic Societies Japan, Tokyo, Japan, 2001).

28 Becker, B., Lechevalier, M. P., Gordon, R. E. \& Lechevalier, H. A. Rapid differentiation between Nocardia and Streptomyces by paper chromatography of whole-cell hydrolysates. Appl. Microbiol. 12, 421-423 (1964).

29 Nakagawa, Y. \& Kawasaki, H. Identification Manual of Actinomycetes (in Japanese), (ed. The Society for Actinomycetes Japan) 83-117 (Business Center for Academic Societies Japan, Tokyo, Japan, 2001).

30 Thompson, J. D., Higgins, D. G. \& Gibson, T. J. CLUSTAL W improving the sensitivity of progressive multiple sequence alignment through sequence weighting, position-specific gap penalties and weight matrix choice. Nucleic Acids Res. 22, 4673-4680 (1994). 